ISSN 2223-7747

www.mdpi.com/journal/plants

Article

\title{
SPL8 Acts Together with the Brassinosteroid-Signaling Component BIM1 in Controlling Arabidopsis thaliana Male Fertility
}

\author{
Shuping Xing ${ }^{1{ }_{*},}$, Vanessa Quodt ${ }^{1}$, John Chandler ${ }^{2}$, Susanne Höhmann ${ }^{1}$, Rita Berndtgen ${ }^{1}$ and \\ Peter Huijser ${ }^{1, *}$
}

1 Department of Comparative Development and Genetics, Max Planck Institute for Plant Breeding Research, Cologne 50829, Germany; E-Mails: hoehmann@mpipz.mpg.de (S.H.); berndtge@mpipz.mpg.de (R.B.)

2 Institute of Developmental Biology, Cologne Biocenter, University of Cologne, Cologne 50674, Germany; E-Mail: john.chandler@uni-koeln.de

* Authors to whom correspondence should be addressed; E-Mails: xing@mpipz.mpg.de (S.X.); huijser@mpipz.mpg.de (P.H.); Tel.: +49-221-5062-172 (S.X.); Fax: +49-221-5062-113 (P.H.).

Received: 22 April 2013; in revised form: 22 May 2013 / Accepted: 18 June 2013 /

Published: 25 June 2013

\begin{abstract}
The non-miR156 targeted SBP-box gene SQUAMOSA PROMOTER BINDING PROTEIN-LIKE 8 (SPL8), plays an important role in Arabidopsis anther development, where its loss-of-function results in a semi-sterile phenotype. Fully male-sterile plants are obtained when a spl8 loss-of-function mutation is introduced into a 35S:MIR156 genetic background, thereby revealing functional redundancy between SPL8 and miR156-targeted SBP-box genes. Here, we show that BIMl, a gene encoding a bHLH protein involved in brassinosteroid signaling and embryonic patterning, functions redundantly with SPL8 in its requirement for male fertility. Although bim1 single mutants displayed a mild fertility problem due to shortened filaments in some flowers, mutation of BIMI significantly enhanced the semi-sterile phenotype of the spl8 mutant. Expression of both SPL8 and BIMI was detected in overlapping expression domains during early anther developmental stages. Our data suggest that in regulating anther development, $S P L 8$ and BIMI function cooperatively in a common complex or in synergistic pathways. Phylogenetic analysis supports the idea of an evolutionary conserved function for both genes in angiosperm anther development.
\end{abstract}


Keywords: anther development; Arabidopsis thaliana; BIM1; brassinosteroid signaling; male fertility; SPL8

\section{Introduction}

In flowering plants, functional development of the male stamen and the female pistil is required for successful sexual reproduction and is affected by many genetic and environmental factors. In the model plant Arabidopsis thaliana, a typical flower has six stamens and one pistil, or gynoecium, formed by two carpels. Organ identity determination, initiation, growth and patterning of these reproductive structures have been widely investigated [1-4]. One of our long-term interests is to understand the molecular mechanisms that underlie the development of the anther, the terminal part of the stamen, which produces the male gametophytes or pollen. According to Sanders et al. [5], the Arabidopsis anther developmental process can be divided into 14 histomorphologically defined stages. Stages 1 to 7 are early developmental stages, predominantly confined to sporogenous cell and anther wall formation in the four corners of the anther. The later postmeiotic Stages 8 to 14, comprise pollen maturation and anther structural adaptations for its release at anthesis.

Many genes are known to be involved in these late anther developmental stages, but relatively few genes are known to act during the earlier stages [6,7], when cell division, commitment and differentiation occur. Among these latter genes, SPOROCYTELESS/NOZZLE (SPL/NZZ) has been characterized in Arabidopsis. Mutation of $s p l / n z z$ leads to the formation of anthers without a proper specification of sporogenous cells and thus, the absence of pollen sacs [8,9]. Interestingly, loss-of-function of both ROXY1 and $R O X Y 2$, two closely-related CC-type glutaredoxin encoding genes, results in a similar phenotype that mainly affects pollen sac formation in the adaxial anther lobes [10]. Recently, SQUAMOSA PROMOTER BINDING PROTEIN-LIKE (SPL) genes, encoding plant-specific SBP-domain transcription factors, have also been reported to play an important role in early anther development. Mutation of SPL8 results in reduced seed set, especially in the first few flowers, where the anthers remain partly or fully devoid of pollen due to an early arrest of sporogenous cell formation in all or some of the anther lobes [11]. A complete loss of pollen production in the anthers of all formed flowers is obtained when the spl8 mutant is combined with a $35 S: M I R 156$ transgene that is able to down-regulate the expression of a set of miR156-targeted SBP-box genes. Such mutant transgenic plants produce fully male sterile organs with anthers lacking all four pollen sacs [12], thereby resembling the spl/nzz mutant phenotype.

Brassinosteroids, a class of plant hormones [13], have also recently been reported to control male fertility by regulating the expression of several key genes involved in anther and pollen development, such as SPL/NZZ, TDF1, AMS, AtMYB103 and MS1 [14]. The basic helix-loop-helix (bHLH) protein BIM1 (BES1-interacting Myc-like1) is a brassinosteroid signaling component involved in regulating BR-induced genes [15] and controls embryo patterning via interaction with the AP2 transcription factors DORNRÖSCHEN (DRN) and DORNRÖSCHEN-LIKE (DRNL) [16]. Here, we describe that BIMI and SPL8 function together to control early anther development. Mutation of BIMI in a spl8 mutant background significantly enhanced the spl 8 semi-sterile phenotype, suggesting that the products of both genes act cooperatively in a common complex or via synergistic pathways to promote Arabidopsis male fertility. 


\section{Results}

\section{1. bim1 Enhances the spl8 Semi-Sterile Phenotype}

As we have reported previously, the Arabidopsis spl8 mutant is semi-sterile, and an additional down-regulation of other, miR156-targeted, $S P L$ genes results in fully sterile plants $[11,12]$. In search of additional genetic factors involved in the spl8 semi-sterile phenotype, we performed crosses between spl8 and some mutants of candidate genes. This way, we selected the bim 1 mutant for further analysis. Morphologically, the bim1 single mutant appeared similar to wild type, except that it had a mild fertility problem, probably due to some flowers producing stamens with shortened filaments (Figure 1b). As for $s p l 8$, the severity of the fertility problem in biml mutant flowers was also found to be dependent on their position in the inflorescence. Whereas the first flowers of the biml mutant primary inflorescence often produced only a few seeds, increasingly more seeds were formed by later-arising flowers (Figure 2a,b). More strikingly, however, seed number was also dramatically reduced in later-arising flowers of spl8 bim 1 double mutants. For example, in the 10th flower of both spl8-1 biml-1 and spl8-1 biml-2 double mutants, the mean seed number was $2.3 \pm 8.0$ and $2.8 \pm 7.5$, respectively, whereas in the single mutants spl8-1, biml-1 and biml-2 this mean was $13.7 \pm 13.0,23.6 \pm 18.8$ and $28.3 \pm 16.6$, respectively, and $50.2 \pm 6.2$ in wild type (Figure $2 \mathrm{~b}$ ). Compared to the respective parental mutant lines, however, the length of stamen filaments showed no obvious further reduction in spl8 biml double mutant flowers (Figure 1c,d; five flowers of each genotype were dissected for comparison). The further reduction in fertility of the spl8 biml double mutant could thus not be ascribed to short filaments. We also tested whether mutations in BIM2 and BIM3, both encoding bHLH proteins closely related to BIM1, would have an effect on spl8 fertility. In contrast to bim1, however, the bim2 and bim3 mutations did not significantly affect spl8 fertility and seed production of the spl8-1 bim 2 bim 3 triple mutant remained comparable to that of the spl8-1 single mutant (Figure 2b). Likewise, seed production in the quadruple mutant spl8-1 bim1 bim2 bim3 resembled that of the spl8-1 bim1 double mutant (data not shown). These data clearly indicate that BIM1, but not BIM2 or BIM3, contribute to the fertility of the spl8 mutant, suggesting that in regulating male fertility, BIMI and SPL8 act in the same or in parallel pathways.

Figure 1. Flower morphology of biml and spl8 mutants at anthesis. To obtain a better view inside the flower, two sepals and one petal were removed. For comparison, the 10th flower was selected. (a) A. thaliana Col-0 wild type, showing dehisced anthers with pollen grains and the stigmatic papillae covered with many pollen grains; (b) bim 1-1, the anthers releasing pollen grains onto the surface of the gynoecium valve due to the short filament; (c) spl8-1, some pollen grains released from anthers touching the stigmatic papillae; (d) spl8-1 bim 1-1, a few pollen grains were deposited on the surface of the valve.

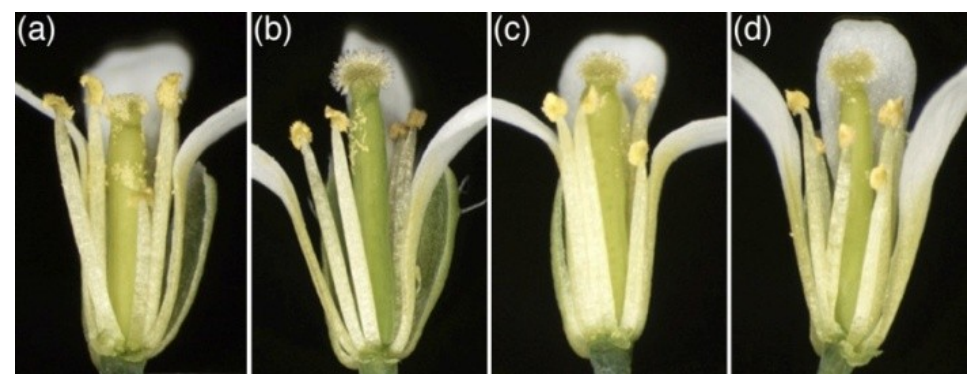


Figure 2. Seed set in bim, spl8 and their combined mutants. (a) Primary inflorescences, excised immediately below the last cauline leaf of $A$. thaliana Col-0 wild type and mutant plants as labeled in the image. (b) Mean seed set per silique from the first, fifth, 10th and 15 th flower, respectively, formed within the primary inflorescences of wild type and mutant plants. Error bars indicate SD $(\mathrm{n}=30)$.

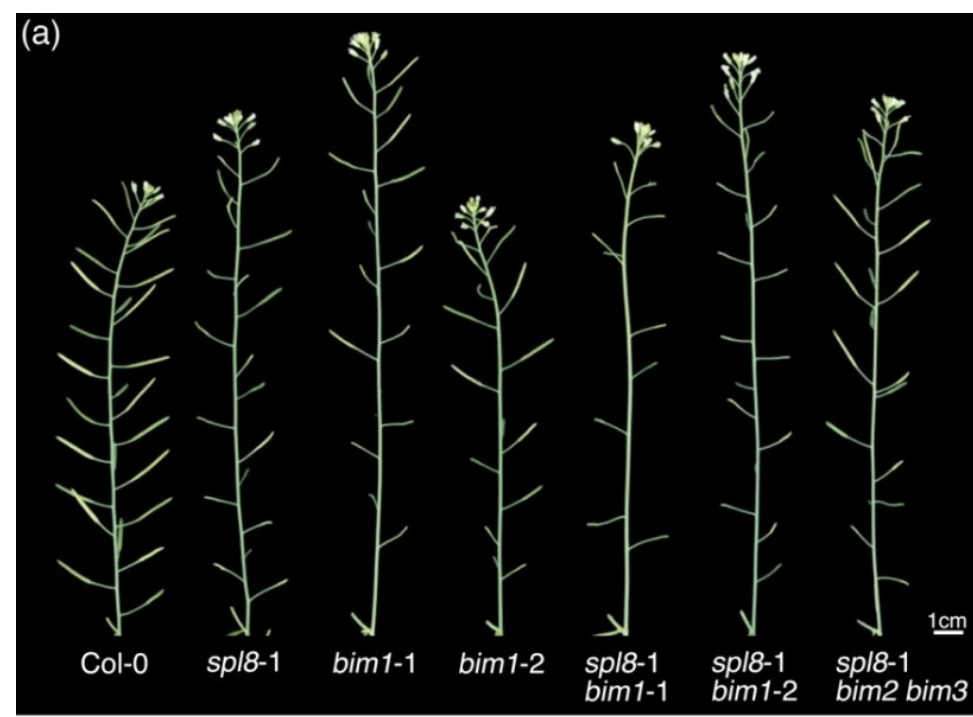

(b)

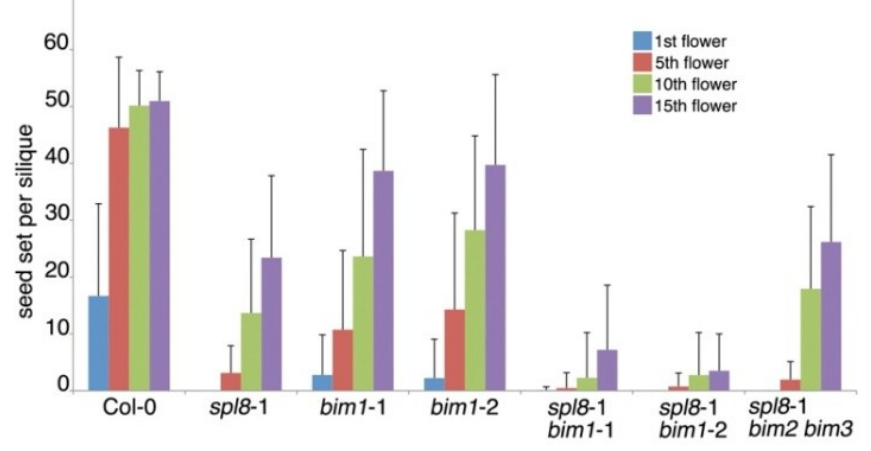

\subsection{Isolation of a New Loss-of-Function Allele for BIMI}

To obtain more solid evidence that the enhancement of the spl8 mutant phenotype in spl8 bim 1 double mutants resulted from the loss of BIMI gene function, we isolated another T-DNA insertion mutant allele from the Wisconsin Dslox Project lines (N850504, Nottingham Arabidopsis Stock Centre). The T-DNA was found to be inserted in the third intron of BIM1, and we named this new mutant allele bim1-3 (Figure 3a). Accordingly, the other two BIM1 alleles used in this study are referred to as bim1-1 (Salk_085924, [15]), bim1-2 (Salk_132178, [16]), and those of BIM2 and BIM3, respectively, as bim2-1 (Salk_074689, [15]) and bim3-1 (Salk_079683, [15]). To test whether bim1-3 represented a loss-of-function mutant, we extracted RNA from the homozygous mutant bim1-3 and wild type plants and performed reverse transcription polymerase chain reaction (RT-PCR) using a primer pair matching exon sequences flanking the T-DNA insertion site (Figure 3a). This showed that whereas a clear band could be amplified from wild type, no amplicon was present for bim1-3, suggesting the latter to be a null allele although it cannot be ruled out that a partial transcript might give rise to residual function. (Figure 3b). Homozygous double mutants generated with this new mutant biml-3 allele and two different mutant spl8 alleles, spl8-1 and spl8-3, respectively, showed fertility defects similar to 
those of the spl8-1 bim1-1 and spl8-1 bim1-2 double mutants mentioned above (data not shown). These results further strengthen the conclusion that loss of BIMI gene function enhances the spl8 semi-sterile phenotype.

Figure 3. Isolation of bim 1, bim 2 and $\operatorname{bim} 3$ mutants. (a) Structures of BIM genes and mutant alleles. The triangle indicates the position of the T-DNA insertion within the gene. P413 and P414 are the two primers used to amplify a portion of the BIMI transcript. (b) Comparison of BIMI expression in inflorescences of $A$. thaliana Col-0 wild type and the bim1-3 mutant by RT-PCR. The RAN3 gene was amplified as a control [10].

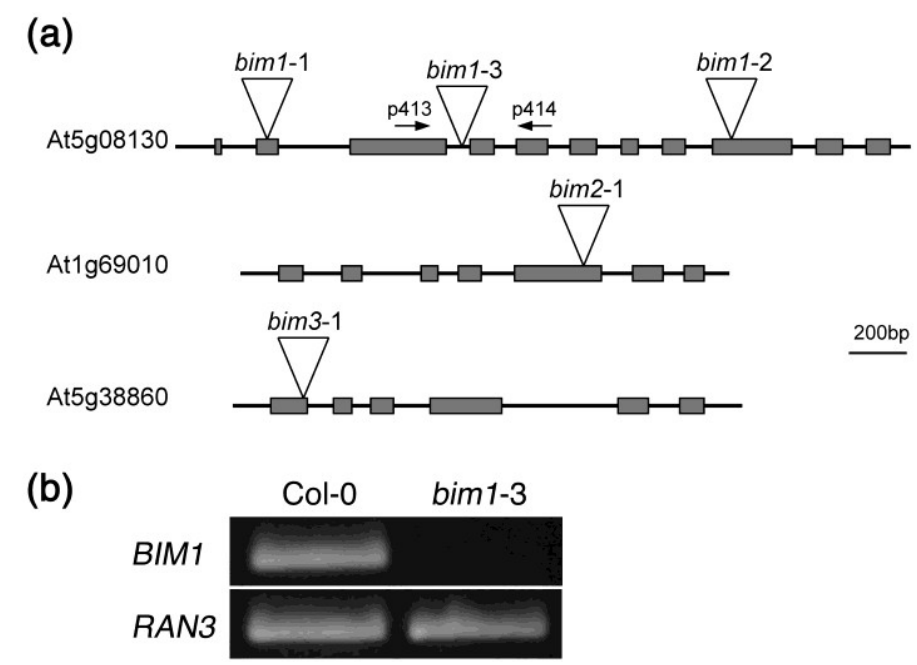

\subsection{Histological Analysis of spl8 bim1 Double Mutant Anthers}

As we concluded above, it appeared unlikely that the strong reduction in fertility of the spl8 bim 1 double mutant flowers could be ascribed to the shortened filaments already observed in bim 1 single mutant flowers (Figure 1a-d). As spl8 single mutant anthers are known to exhibit well-defined developmental defects, we thus focused on the developing double mutant anthers. For proper comparison we always selected the 10th flower from primary inflorescences of wild type, spl8-1, bim 1-1 and spl8-1 bim1-1 mutant plants, and prepared semi-thin cross-sections from their anthers. At an early anther developmental stage, e.g., Stage 5, wild type showed distinct anther wall cell layers surrounding the pollen mother cells (PMCs) at each of the four loculi (Figure 4a). At a post-meiotic stage, e.g., Stage 8, these loculi had developed into four pollen sacs containing many pollen grains (Figure 4b). Similar developmental stages in both the spl8-1 (Figure 4c,d) and biml-1 (Figure 4e,f) single mutant anthers showed a comparable degree of tissue differentiation. Occasionally however, an adaxially-positioned loculus failed to develop in the spl8-1 mutant, in which case, sporogenous cells did not appear to be specified (Figure 4c), and thus no functional pollen sac formed (Figure 4d). Clearly, comparably staged spl8-1 bim 1-1 double mutant anthers exhibited more severe developmental anomalies. Sporogenous cell formation appeared to be disturbed (Figure 4g), particularly in the adaxial loculi, resulting in anthers lacking pollen sacs at these positions (Figure 4h). In some cases, the PMCs appeared not to have undergone meiosis and later degenerated in the pollen sacs (Figure $4 \mathrm{~h}$ ). Given these observations, we conclude that BIMI and SPL 8 are both required for early anther development. 
Figure 4. Comparative histological structures of anthers. Semi-thin cross-sections through Arabidopsis wild type (a,b), spl8-1 (c,d), bim1-1 (e,f) and spl8-1 bim1-1 (g,h) anthers from the 10th flower were stained with toluidine blue. (a), (c), (e), (g) Anthers at Stage 5, the pollen mother cells (PMCs) are present in the center and are surrounded by distinct anther wall layers in the wild type, spl8-1 and bim 1-1 mutants. Note that the lower right locule of this particular spl8-1 anther and all four loculi of sp8-1 biml-1 anthers lack typical PMCs (stars). (b), (d), (f), (h) Anthers at Stage 8. Pollen grains were formed in pollen sacs of wild type, spl8-1 and bim 1-1 mutants, however, the number of the pollen grains in each pollen sac and the number of pollen sacs was reduced in the spl8-1 mutant (d), and this phenotype was further enhanced when spl8 was combined with biml. The abaxial PMCs degenerated and non-functional pollen grains were formed in this particular spl8-1 bim1-1 anther (h). pmc, pollen mother cell; v, vascular bundle. $\mathrm{Bar}=50 \mu \mathrm{m}$.
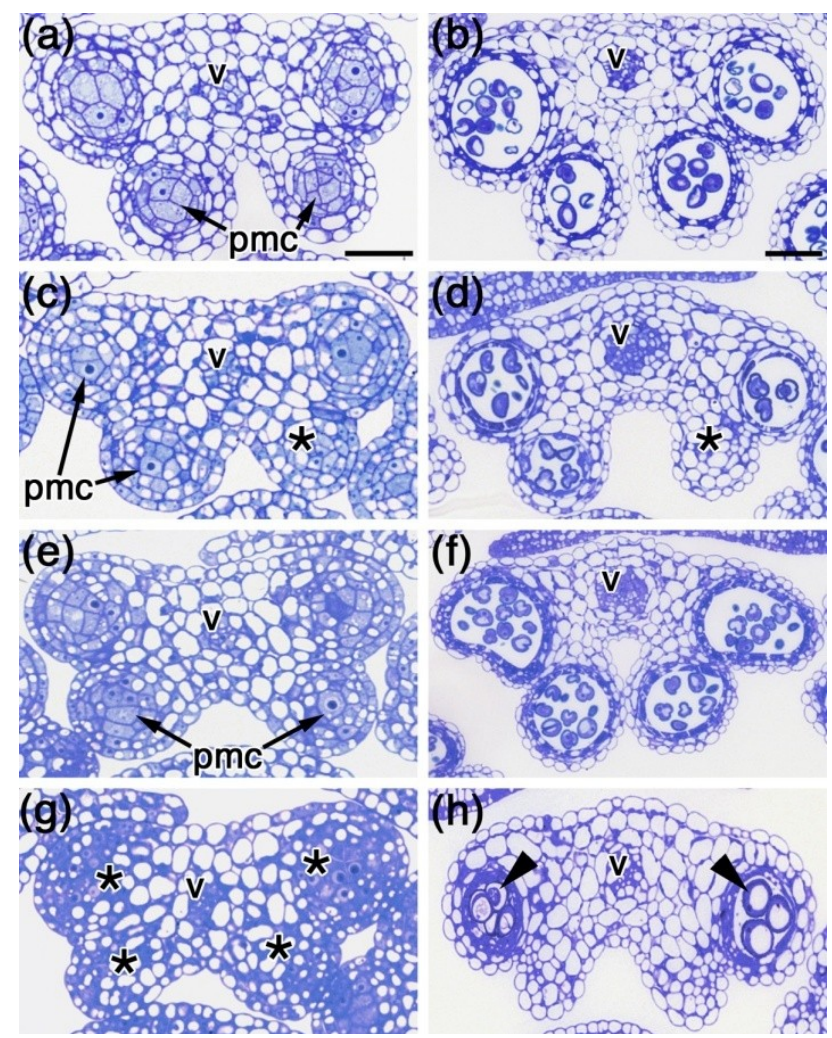

\subsection{Expression of BIM1 in Anthers}

As the genetic and histological data revealed the function of $B I M I$ to be required for proper anther development, we performed in situ hybridization experiments to investigate BIMI spatial and temporal expression in anthers. For comparison, we hybridized a SPL8 probe in parallel. BIMI expression was already clearly detectable in early Stage 3 anthers, when archesporial cell divisions in the four corners start to give rise to the sporogenous tissue [5]. In this early stage, the BIMI hybridization signal spread over the entire anther and gynoecium cross-section (Figure 5a), whereas SPL 8 hybridization appeared only in the four corners of the anthers and was weakly detectable in medial regions of the gynoecium (Figure 5d). At the subsequent developmental Stage 4, when more sporogenous cells had formed, BIM1 expression started to resemble broadly that of SPL8, i.e., more confined to the four corners of the 
anthers and the medial regions of the gynoecium (Figure 5b,e). At Stage 6, just before the PMCs enter meiosis, BIM1 and SPL8 displayed a very similar expression domain largely covering the PMCs, their surrounding anther cell wall layers and the carpel marginal meristems of the gynoecium (Figure 5c,f). These data indicate that BIMI and SPL 8 expression largely overlap in the early anther and provide further evidence that these genes might act together in regulating anther development.

Figure 5. In situ hybridization analysis of BIMI and SPL8 in early flower buds from primary inflorescences of Arabidopsis wild type plants. Cross-sections through wild type early flowers at several stages were analyzed for BIMI (a-c) and SPL8 (d-f) expression. (a,d) Cross-sections through the flower buds containing Stage 3 anthers, showing BIMI expression in the anthers and gynoecium, and distinct SPL8 expression mainly in the four corners of the anthers. (b,e) At anther Stage 4, both BIMI and SPL8 are expressed in all four lobes of the anthers, in both sporogenous cells and somatic cell layers. (c,f) Before meiosis, at anther Stage 6, expression of both genes is mainly confined to PMCs and anther walls. an, anther; g, gynoecium. Bars $=50 \mu \mathrm{m}$.

(a)
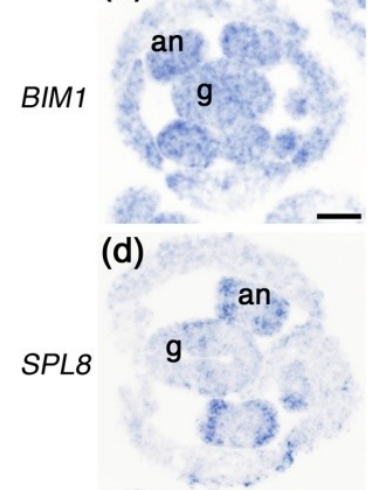

(b)

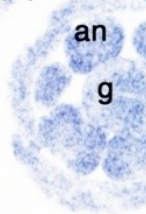

(e)

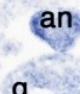

g (c)

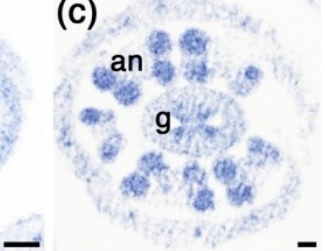

(f)

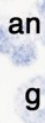

\subsection{Both SPL8 and BIM1 Are Conserved in Dicot and Monocot Plants}

The anther is an evolutionary well-conserved organ in flowering plants and its complexity and critical importance for fertility imply that the basic molecular genetic mechanisms underlying its development are also conserved.

Both SPL8 and BIM1 represent evolutionary diversified and ancient families of transcription factors in plants [17-19]. To determine whether other dicots as well as monocots possess highly similar proteins with probably functional homologous properties, we performed a BLAST search of the NCBI GenBank database. In addition, we queried the Arabidopsis SPL8 and BIM1 proteins against the Plant Transcription Factor Database for predicted orthologs (PlantTFDB, [20]). Representing species with available whole genome sequence data, we chose the dicots poplar (Populus trichocarpa) and tomato (Solanum lycopersicum), and the monocots rice (Oryza sativa var. japonica) and Brachypodium distachyon. Multiple alignments and phylogenetic analysis of the sequences obtained, showed the unique Arabidopsis SPL8 gene to be represented by two closely related and probably paralogous genes in each of the other species (Figure 6a). Arabidopsis BIMI also appeared to be represented by two copies in both other dicot species, as were the related Arabidopsis BIM2 and BIM3 genes, with the exception of only one BIM3-like copy being identified in tomato (Figure 6b). 
Interestingly, the BIM-related monocot proteins clustered within a separate group and appeared to be somewhat more related to BIM2 and BIM3. However, a more direct comparison of the monocot proteins suggested an overall higher structural similarity to BIM1, with an extended $N$-terminal domain that was lacking in BIM2 and BIM3. Comparing the respective genomic loci further supported the idea that the identified BIM-related monocot genes and Arabidopsis BIMI probably share a more ancestral intron-exon structure (Figure 6c).

In addition to Arabidopsis, evolutionary diverged flowering plants express proteins highly similar to SPL8 and BIM1, suggesting a role for these proteins in anther development in other dicots and possibly also in monocots.

Figure 6. Phylogenetic analysis of SPL8- (a) and BIM-like (b) proteins. The entire protein sequences encoded by Arabidopsis (AtSPL8, AtBIM), poplar (PtSBP, PtBIM), tomato (SlySBP, SlyBIM), rice (OsSPL, OsBIM), Brachypodium (BdiSBP, BdiBIM) and moss (PpSBP, PpBIM) genes were aligned using ClustalW. The phylogram was constructed using the neighbor-joining algorithm using Physcomitrella patens-related sequences for rooting. Bootstrap values $>50 \%$ are shown adjacent to the respective nodes. Database accession numbers are shown after each protein. The genomic organization of the Arabidopsis BIM1, BIM2 and BIM3 loci are compared to those of the two most closely related $B I M$ genes in rice $(\mathbf{c})$.

(a)

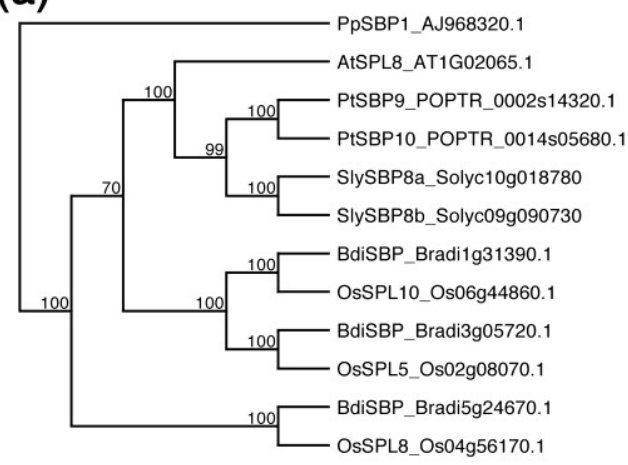

(b)

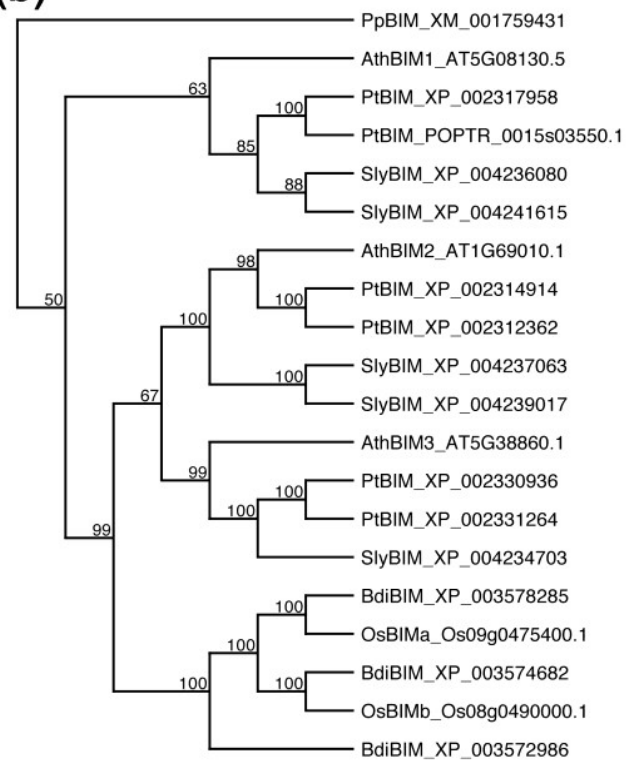

(c)

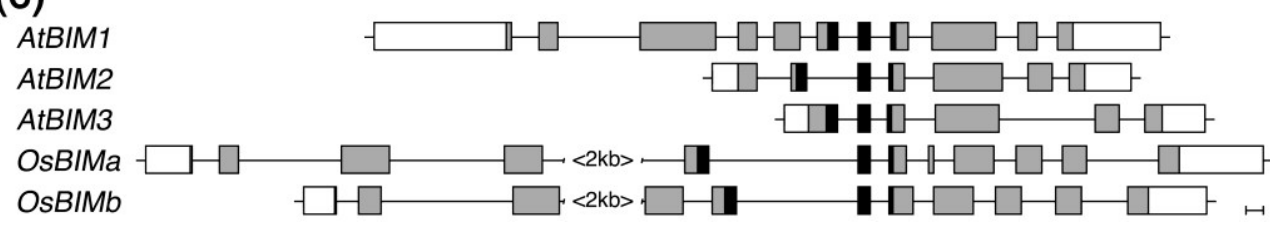

\section{Discussion}

In our growth conditions, generally only the first few flowers formed in spl8 mutant primary inflorescences showed strongly reduced seed set. However, the number of flowers with severely affected 
fertility dramatically increased in the primary inflorescence of the spl8 biml double mutant (Figure 2). Our results showed that the bim 1 mutation enhanced the spl8 mutant phenotype with respect to anther histogenesis, suggesting that $B I M 1$, similar to $S P L 8$, regulates early anther development. Furthermore, in situ hybridization revealed a large degree of overlap in the temporal and spatial expression patterns of both genes during early stages of anther development, implying a possible interaction in controlling sporogenous cell formation and the establishment of parietal cell layers in anther patterning.

BIM1 has been identified as a putative SPL8-interacting protein in a yeast two-hybrid screen using SPL8 as bait [21]. It thus appears possible that SPL8 and BIM1, both known to be DNA-binding transcription factors, form a complex to regulate shared target genes in early anther development. This raises the interesting question of whether SPL8 or other SBP-box genes are involved in brassinosteroid (BR) signaling in controlling anther development. A previous study has shown that BES1 (bri1-Ethylmethane Sulphonate suppressor 1), a key component of the BR signaling pathway, interacts with BIM1 to regulate BR-responsive genes [15]. Another study indicates that BES1 directly binds to the promoter regions of several key genes in anther and pollen development [14]. Therefore, it is tempting to speculate that SPL8 might regulate early anther development as a component of a BES1-BIM1-SPL8 complex. Other SBP-box genes such as miR156-targeted SPLs, with functional redundancy to SPL8 [12], might also join the same pathway to regulate early anther development. The generation and analysis of double mutants between spl8 and other mutants in BR biosynthesis and signaling will help to clarify whether BIM1 indeed provides a unique link between SPL8 and the BR pathway.

Our preliminary phylogenetic analysis showed that closely related and putative orthologs of SPL8 and BIMI are identifiable in both monocotyledonous and dicotyledonous plants (Figure 6). However, whereas dicots also appear to possess clear BIM2 and BIM3 counterparts, monocot BIM-like genes appear to form a separate clade. We showed that in combination with SPL8, BIM1 but not BIM2 or BIM3 affected male fertility. A major difference between these three closely related BIM-like proteins is the presence of a $N$-terminal extension to the bHLH region in BIM1. Differences also exist in the intron-exon structures of the respective genes. Interestingly, this extension is conserved in the monocot BIM sub-family members. Taken together, and in the context of the evolutionary conserved process of anther development, this might imply that a possible interaction between functional SPL8 and BIM1 homologs is expected to be conserved in both mono- and dicotyledonous plants.

In addition to BR, the phytohormone auxin has also been demonstrated to play a role in anther dehiscence, pollen maturation and filament elongation [22]. Auxin and BR act synergistically, for instance via a BR-mediated degradation of repressors of some auxin activated genes [23]. However, to date there is little support for auxin functioning in early anther development, although some auxin biosynthesis genes are expressed in early anthers [24]. Auxin is known to play an important role in leaf initiation and development and thus probably also in the early development of leaf-derived organs such as anthers and carpels. Moreover, BIM1 interacts with DRN/DRNL, acting directly downstream of auxin responses and is involved in embryonic patterning and lateral organ formation [25,26]. Our own recent data indicate that SBP-box genes such as SPL8 affect gynoecium development most probably via a link with auxin (Xing and Huijser, unpublished data). Auxin and BR signaling pathways controlling anther development, might thus cross-talk through both SPL8 and BIM1 genes. 


\section{Experimental Section}

\subsection{Plant Materials and Growth Conditions}

Arabidopsis thaliana ecotype Columbia (Col-0; NASC N1092) was used as wild type and all mutants were in the Col-0 background. The mutant alleles bim1-1 (Salk_085924, [15]), bim1-2 (Salk_132178, [16], bim2-1 (Salk_074689, [15] and bim3-1 (Salk_079683, [15] and spl8 single mutants [11] have been described previously. Seeds for bim1-3 (N850504) were obtained from the Nottingham Arabidopsis Stock Centre. For identifying homozygouss of bim1-3 mutants, a T-DNA Left border primer (5'-AACGTCCGCAATGTGTTATTAAGTTGTC-3'), and two gene-specific primers (5'-TTGCAGCTCGTACGCCGCTGCATCAAGC-3' and 5'-TCTTCCTGTGAACTTCCTTTT GCTGACC-3') were used. Prior to sowing, seeds were imbibed and stratified for 2 days in the dark at $4{ }^{\circ} \mathrm{C}$. Plants were cultivated on prefertilized soil mixture (Type ED73; Werkverband) and grown in the greenhouse at $21-23{ }^{\circ} \mathrm{C}$ under long-day conditions (16 h light).

\subsection{Histology and Microscopy}

Embedding and sectioning of flowers as well as seed set determination were performed according to Xing et al. [12].

\subsection{Semi-Quantitative RT-PCR}

Inflorescence tips without open flowers of wild type and the bim1-3 mutant were harvested from 30-day-old plants. RNA isolation and semi-quantitative RT-PCR were performed as described by Xing and Zachgo [10]. The position of the two primers for amplifying part of BIMI cDNA transcripts is shown in Figure 3a (P413: 5'-TGTCACCACCAATGATGTTCAATGC-3' and P414: 5'-TCATCTTCC TGTGAACTTCCTTTTGC-3').

\subsection{In Situ RNA Hybridization}

RNA in situ hybridization was performed as described previously [27]. A SPL 8 antisense probe was prepared according to the method described by Xing et al. [12]. To generate BIMI antisense probes and to avoid cross-hybridization with other BIM gene transcripts, the cDNA sequences of a $220 \mathrm{bp} 5$ ' end fragment with primers (5'-GTAGTCACTTGAAAACCCATGATTTTC-3' and 5'-GCTCgtaatacgactcac tatagggeTGGTGGTGACAACTCCGGCTTAG-3') and a 540 bp 3' end fragment with primers (5'-GATCATGAAGTTCGTGAACCGGTTTCTCG-3' and 5'-GCTCgtaatacgactcactatagggeTCGTTC ATGCGCTTTGGTTTAG-3'), were amplified as templates, respectively. T7 RNA polymerase (Roche Diagnostics GmbH, Mannheim, Germany) was used for in vitro transcription.

\subsection{Phylogenetic Tree Construction}

Multiple alignments of amino acid sequences were generated by the program ClustalW of the MacVector 9.5.2 software package (Accelrys Ltd., Cambridge, UK) using the Gonnet matrix with an open gap penalty of 10 and an extend gap penalty of 0.1 . The phylograms based on these alignments were constructed using the neighbor-joining algorithm available in the same software package. A 
bootstrap analysis with 10,000 repetitions was performed with random tiebreaking and gaps in the multiple alignments ignored.

\section{Conclusions}

Based on the results in this study, we conclude that BIM1 provides a newly identified early anther gene function together with SPL8 in the same or in a parallel pathway to control anther development by regulating sporogenous cell formation and the differentiation and patterning of anther wall cell layers. In addition, BIM1 is a component of the brassinosteroid signaling pathway. Thus, SPL8 and other miR156-targeted SPL genes might join the BR signaling via a link with BIM1 in regulating the early events of anther development.

\section{Acknowledgments}

We thank Maria Salinas for help with the semi-quantitative RT-PCR experiments (Max Planck Institute for Plant Breeding Research, Cologne, Germany), and Yanhai Yin (Iowa State University, Department of Genetics, Development and Cell Biology) for providing bim1-1 seeds. This project was funded by the Deutsche Forschungsgemeinschaft through SFB572.

\section{Conflict of Interest}

The authors declare no conflict of interest.

\section{References}

1. Coen, E.S.; Meyerowitz, E.M. The war of the whorls: Genetic interactions controlling flower development. Nature 1991, 353, 31-37.

2. Bowman, J.L.; Baum, S.F.; Eshed, Y.; Putterill, J.; Alvarez, J. Molecular genetics of gynoecium development in Arabidopsis. Curr. Top. Dev. Biol. 1999, 45, 155-205.

3. Scott, R.J.; Spielman, M.; Dickinson, H.G. Stamen structure and function. Plant Cell 2004, 16, S46-S60.

4. Causier, B.; Schwarz-Sommer, Z.; Davies, B. Floral organ identity: 20 years of ABCs. Semin. Cell Dev. Biol. 2010, 21, 73-79.

5. Sanders, P.M.; Bui, A.Q.; Weterings, K.; McIntire, K.N.; Hsu, Y.C.; Lee, P.Y.; Truong, M.T.; Beals, T.P.; Goldberg, R.B. Anther developmental defects in Arabidopsis thaliana male-sterile mutants. Sex. Plant Reprod. 1999, 11, 297-322.

6. Ma, H. Molecular genetic analyses of microsporogenesis and microgametogenesis in flowering plants. Annu. Rev. Plant Biol. 2005, 56, 393-434.

7. Xing, S.; Salinas, M.; Huijser, P. New players unveiled in early anther development. Plant Signal. Behav. 2011, 6, 934-938.

8. Yang, W.C.; Ye, D.; Xu, J.; Sundaresan, V. The SPOROCYTELESS gene of Arabidopsis is required for initiation of sporogenesis and encodes a novel nuclear protein. Genes Dev. 1999, 13, 2108-2117. 
9. Schiefthaler, U.; Balasubramanian, S.; Sieber, P.; Chevalier, D.; Wisman, E.; Schneitz, K. Molecular analysis of NOZZLE, a gene involved in pattern formation and early sporogenesis during sex organ development in Arabidopsis thaliana. Proc. Natl. Acad. Sci. USA 1999, 96, 11664-11669.

10. Xing, S.; Zachgo, S. ROXY1 and ROXY2, two Arabidopsis glutaredoxin genes, are required for anther development. Plant J. 2008, 53, 790-801.

11. Unte, U.S.; Sorensen, A.M.; Pesaresi, P.; Gandikota, M.; Leister, D.; Saedler, H.; Huijser, P. SPL8, an SBP-box gene that affects pollen sac development in Arabidopsis. Plant Cell 2003, 15, 1009-1019.

12. Xing, S.; Salinas, M.; Höhmann, S.; Berndtgen, R.; Huijser, P. miR156-targeted and nontargeted SBP-box transcription factors act in concert to secure male fertility in Arabidopsis. Plant Cell 2010, 22, 3935-3950.

13. Kutschera, U.; Wang, Z.Y. Brassinosteroid action in flowering plants: A darwinian perspective. J. Exp. Bot. 2012, 63, 3511-3522.

14. Ye, Q.; Zhu, W.; Li, L.; Zhang, S.; Yin, Y.; Ma, H.; Wang, X. Brassinosteroids control male fertility by regulating the expression of key genes involved in Arabidopsis anther and pollen development. Proc. Natl. Acad. Sci. USA 2010, 107, 6100-6105.

15. Yin, Y.; Vafeados, D.; Tao, Y.; Yoshida, S.; Asami, T.; Chory, J. A new class of transcription factors mediates brassinosteroid-regulated gene expression in Arabidopsis. Cell 2005, 120, 249-259.

16. Chandler, J.W.; Cole, M.; Flier, A.; Werr, W. BIM1, a bHLH protein involved in brassinosteroid signalling, controls Arabidopsis embryonic patterning via interaction with DORNRÖSCHEN and DORNRÖSCHEN-LIKE. Plant Mol. Biol. 2009, 69, 57-68.

17. Riese, M.; Höhmann, S.; Saedler, H.; Münster, T.; Huijser, P. Comparative analysis of the SBP-Box gene families in $P$. patens and seed plants. Gene 2007, 401, 28-37.

18. Salinas, M.; Xing, S.; Höhmann, S.; Berndtgen, R.; Huijser, P. Genomic organization, phylogenetic comparison and differential expression of the SBP-box family of transcription factors in tomato. Planta 2012, 235, 1171-1184.

19. Pires, N.; Dolan, L. Origin and diversification of basic-helix-loop-helix proteins in plants. Mol. Biol. Evol. 2010, 27, 862-874.

20. Zhang, H.; Jin, J.P.; Tang, L.; Zhao, Y.; Gu, X.C.; Gao, G.; Luo, J.C. PlantTFDB 2.0: update and improvement of the comprehensive plant transcription factor database. Nucleic Acids Res. 2011, 39, D1114-D1117.

21. Zhang, Y. The SBP-box gene SPL 8 affects reproductive development and gibberellin response in Arabidopsis. Ph.D. Thesis, University of Cologne, Cologne, Germany, 2005.

22. Cecchetti, V.; Altamur, M.M.; Falasca, G.; Costantino, P.; Cardarelli, M. Auxin regulates Arabidopsis anther dehiscence, pollen maturation, and filament elongation. Plant Cell 2008, 20, 1760-1774.

23. Vert, G.; Walcher, C.L.; Chory, J.; Nemhauser, J.L. Integration of auxin and brassinosteroid pathways by Auxin Response Factor 2. Proc. Natl. Acad. Sci. USA 2008, 105, 9829-9834.

24. Sundberg, E.; Østergaard, L. Distinct and dynamic auxin activities during reproductive development Cold Spring Harb. Perspect. Biol. 2009, 1, a001628. 
25. Kirch, T.; Simon, R.; Grünewald, M.; Werr, W. The DORNRÖSCHEN/ENHANCER OF SHOOT REGENERATION1 gene of Arabidopsis acts in the control of meristem cell fate and lateral organ development. Plant Cell 2003, 15, 694-705.

26. Chandler, J.W.; Cole, M.; Flier, A.; Grewe, B.; Werr, W. The AP2 transcription factors DORNRÖSCHEN and DORNRÖSCHEN-LIKE redundantly control Arabidopsis embryo patterning via interaction with PHAVOLUTA. Development 2007, 134, 1653-1662.

27. Xing, S.; Rosso, M.G.; Zachgo, S. ROXY1, a member of the plant glutaredoxin family, is required for petal development in Arabidopsis thaliana. Development 2005, 132, 1555-1565.

(C) 2013 by the authors; licensee MDPI, Basel, Switzerland. This article is an open access article distributed under the terms and conditions of the Creative Commons Attribution license (http://creativecommons.org/licenses/by/3.0/). 